\title{
Stopping Powers of Protons in Biological Human Body Substances
}

\author{
Hemalata Singh ${ }^{1, *}$, S. K. Rathi ${ }^{1}$, A. S. Verma ${ }^{2}$ \\ ${ }^{1}$ Department of physics, B. S. A. College, Mathura, India, 281004 \\ ${ }^{2}$ Department of physics, Banasthali Vidyapith, Rajasthan, 304022 \\ *Corresponding Author: ajay_phy@rediffmail.com
}

Copyright (C) 2013 Horizon Research Publishing All rights reserved.

\begin{abstract}
In this paper we present a simple method for the calculation of mass stopping power (in $\mathrm{MeV} \mathrm{cm} / \mathrm{g}$ ) of protons from $0.5 \mathrm{Mev}$ to $200 \mathrm{MeV}$ energy range in biological human body substances such as water, bone, muscle and tissue. The proposed relations have been described in terms of energy of proton and mean $(<\mathrm{Z} / \mathrm{A}>)$ of atomic number $(\mathrm{Z})$ and atomic weight (A). A fairly good agreement has been found between our simplified values for mass stopping power of protons for water, bone, muscle and tissue and that of Mukherjee calculated values.
\end{abstract}

Keywords Stopping Power, Atomic Weight, Atomic Number

\section{Introduction}

Information on stopping power is essential in many fields involving radiation. Their accuracy may critically affect calculations, measurements and interpretation of experiments. Research concerning the stopping power has taken the position of the basic theme in the fields of ion-matter interactions for a long time. Despite the long history of stopping power research, the current knowledge, both experiment and theoretical, is far from being complete, and is often inadequate for the determination of stopping power values of a variety of materials and for a wide range of particle energies.

Energy deposition models of electrons, positrons and protons passing through matter are necessary for several radiological and dosimetric applications. The physical aspects of the energy loss process of low energy protons in human body substances are of great interest for trace element analysis by proton induced X-rays. The stopping power of the protons, which is directly related to the linear energy transfer, must be well estimated in order to determine the proton dose distribution in the irradiated tissue accurately [1,2]. In this study, we propose a method to obtain the mass stopping power for protons at energy
$(0.5-200 \mathrm{MeV})$. Results obtained by this procedure are compared with the available data.

\section{Theory, Results and Discussion}

The stopping power has been used in Monte Carlo simulations of electron transport relevant to electron probe microanalysis [3-5]. The Bethe stopping power equation [6-8] has been used extensively for energies where it is expected to be valid, but there is a scarcity of data at lower energies. The classical Bethe theory on the interaction of electrons with matter has been based on the Born approximation. Stopping powers calculated from the Bethe equation are available from a NIST database for electron energies of $10 \mathrm{keV}$ and above [9].

Several empirical relations are given in literature to simplify the expressions for stopping power. One of them is due to Sargent [10]. His expression for the rate of change of velocity of low energy $(<100 \mathrm{keV})$ electrons in Aluminum is

$$
-\mathrm{d} \beta / \mathrm{dx}=2.2 / \beta^{3}
$$

$\beta$ is the ratio of velocity of electron to velocity of light and thickness $\mathbf{x}$ is expressed in cms.

Heitler [11] established a relation for collision stopping power which is valid for somewhat higher energies also. This is given by

$$
-(\mathrm{dE} / \mathrm{dx})_{\text {ion }}=\xi \ln (\eta \mathrm{W})
$$

$\mathrm{W}$ is the total energy expressed in $\mathrm{MeV}$ and $\xi$ and $\eta$ are the constants which differ from material to materials.

Batra and Sehgal [12-14] have studied the total stopping power of electron and positron by the product of two functions. These functions must depend on the kinetic energy $(\mathrm{T})$ of electron or positrons and the atomic number $(\mathrm{Z})$ of the material. According to them these equations were valid for energies up to $5.0 \mathrm{MeV}$ for materials of atomic number up to 92 .

For $\mathrm{T} \leq 0.25 \mathrm{MeV}$

$$
\begin{gathered}
(-1 / \rho * \mathrm{dE} / \mathrm{dx})_{\mathrm{Tot}}^{ \pm}=\left(\mathrm{M}_{1} \mathrm{Z}+\mathrm{C}_{1}\right) \mathrm{F}^{ \pm}(\gamma) \\
\mathrm{F}^{+}(\gamma)=\gamma^{2.4} / \gamma^{(1.9-1)}
\end{gathered}
$$




$$
\mathrm{F}^{-}(\gamma)=\gamma^{2.56} /\left(\gamma^{2}-1\right)
$$

Where $\rho$ is the density of target material and $\gamma$ is the total energy of electron or positron in electron mass unit. $M_{1}$ and $\mathrm{C}_{1}$ are constants.

For $0.25 \mathrm{MeV} \leq \mathrm{T} \leq 5.0 \mathrm{MeV}$

$$
(-1 / \rho * d E / d x)^{ \pm}=\left(M_{2} Z+C_{2}\right)\left[\gamma^{2} / \gamma^{a+z+b \pm}-1\right]
$$

Superscripts \pm represents for positron and electron respectively. $\mathrm{M}_{2}, \mathrm{C}_{2}, \mathrm{a}^{ \pm}$and $\mathrm{b}^{ \pm}$are constants.

Pal et al [15] developed a similar formula on the lines of Batra and Sehgal [12-14] but with different set of constants,

$$
-1 / \rho(\mathrm{d} \gamma / \mathrm{dx})^{ \pm}=(\mathrm{MZ}+\mathrm{N})\left(\mathrm{P}_{0}^{ \pm}+\mathrm{P}_{1}^{ \pm} \gamma\right)
$$

$\mathrm{P}_{\mathrm{n}}^{ \pm}=\mathrm{A}_{\mathrm{n}}{ }^{ \pm}+\mathrm{B}_{\mathrm{n}}^{ \pm} \mathrm{Z}+\mathrm{C}_{\mathrm{n}}^{ \pm} \mathrm{Z}^{2}$, where A, B and C are constants and $n=0 \& 1$. This relation holds well from 5 to $1000 \mathrm{meV}$.

\section{Proposed Relation and Calculated Values for Stopping Power}

Recently, Tanuma et al [16] has calculated electron stopping power for 31 elemental solids. These stopping powers are determined with an algorithm previously used for the calculation of electron inelastic mean free paths and from energy loss functions derived from experimental optical data. The stopping power calculations are valid for electron energies between $100 \mathrm{eV}$ and $30 \mathrm{keV}$. Through exponential fitting, we have able to find out empirical relations for total stopping power from $0.5 \mathrm{MeV}$ to $200 \mathrm{MeV}$. In general the exponential fitting is a simple method for searching out any empirical relation. However, the ration for total stopping power should be simple enough to get easily integrable. One can infer from equations ( 1 to 5 ) that the total stopping powers of electrons as well as that of positrons depend not only upon the incident kinetic energy of these particles, but also on the nature of the material through which they traverse. It was noticed that the dependence of total stopping power on incident kinetic energy could only be met through the use of some suitable power function. We have plotted graphs between available stopping power values vs $\left\langle\frac{Z}{A}\right\rangle E^{0.05}$ and data are decreasing exponentially. Which are presented in following figures 1 to 4 . Using this idea we have been able to find analytically convenient and simple empirical relation for mass stopping power of protons in energy regions 0.5 to $200 \mathrm{MeV}$ by the following relation,

mass stopping power $=y_{o}+A_{1} e^{\left(-\frac{x}{t_{1}}\right)}$

where $y_{0}, A_{1}$ and $t_{1}$ are constants. The value of ' $x=\left\langle\frac{Z}{A}\right\rangle E^{0.05}$, depends on atomic number (Z), atomic weight (A) and energy (E). The values of constants are presented in table 1 .

Table 1. Values of constants for protons

\begin{tabular}{ccccc}
\hline Materials & $\mathrm{y}_{\mathrm{o}}$ & $\mathrm{A}_{1}$ & $\mathrm{t}_{1}$ & $\mathrm{R}^{2}$ \\
\hline Water & 0 & $3.49705 \times 10^{8} \pm 3.877 \times 10^{7}$ & $0.03981 \pm 3.14697 \times 10^{-4}$ & 0.99906 \\
Muscle & 0 & $2.82992 \times 10^{8} \pm 3.468 \times 10^{7}$ & $0.0396 \pm 3.50469 \times 10^{-4}$ & 0.99881 \\
Bone & 0 & $1.96457 \times 10^{8} \pm 3.624 \times 10^{7}$ & $0.03886 \pm 5.2698 \times 10^{-4}$ & 0.99711 \\
Tissue & 0 & $3.6275 \times 10^{8} \pm 4.290 \times 10^{7}$ & $0.03904 \pm 3.29012 \times 10^{-4}$ & 0.99894
\end{tabular}

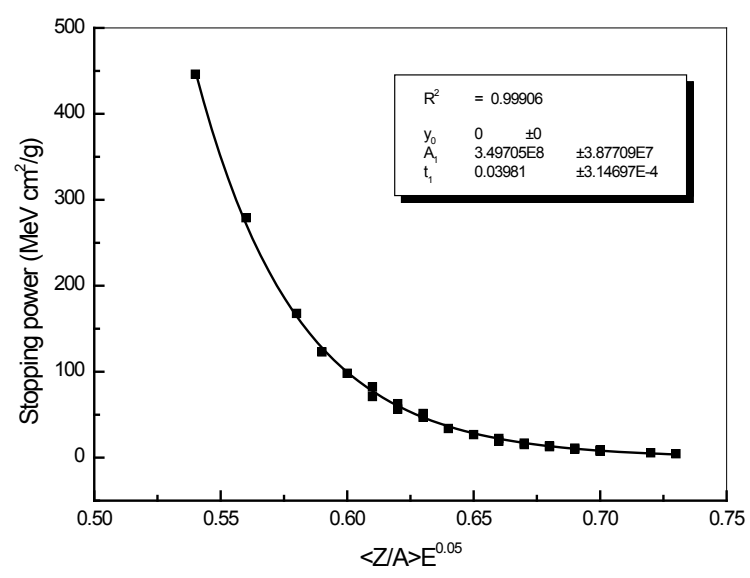

Figure 1. In this plot of mass stopping power (for protons) and $\left\langle\frac{Z}{A}\right\rangle E^{0.05}$ of Water lie on a decreasing exponentially line. In this figure all values 
are taken from Ref. [2].

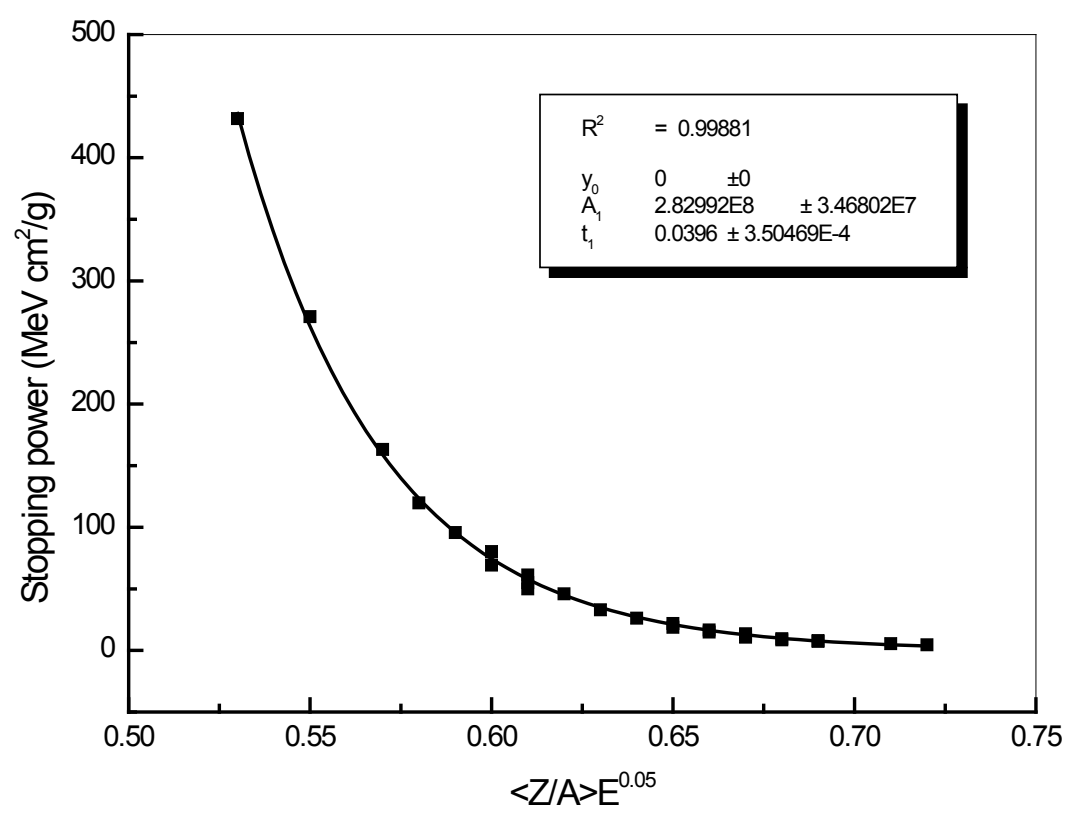

Figure 2. In this plot of mass stopping power (for protons) and $\left\langle\frac{Z}{A}\right\rangle E^{0.05} \quad$ of Muscle lie on a decreasing exponentially line. In this figure all values are taken from Ref. [2].

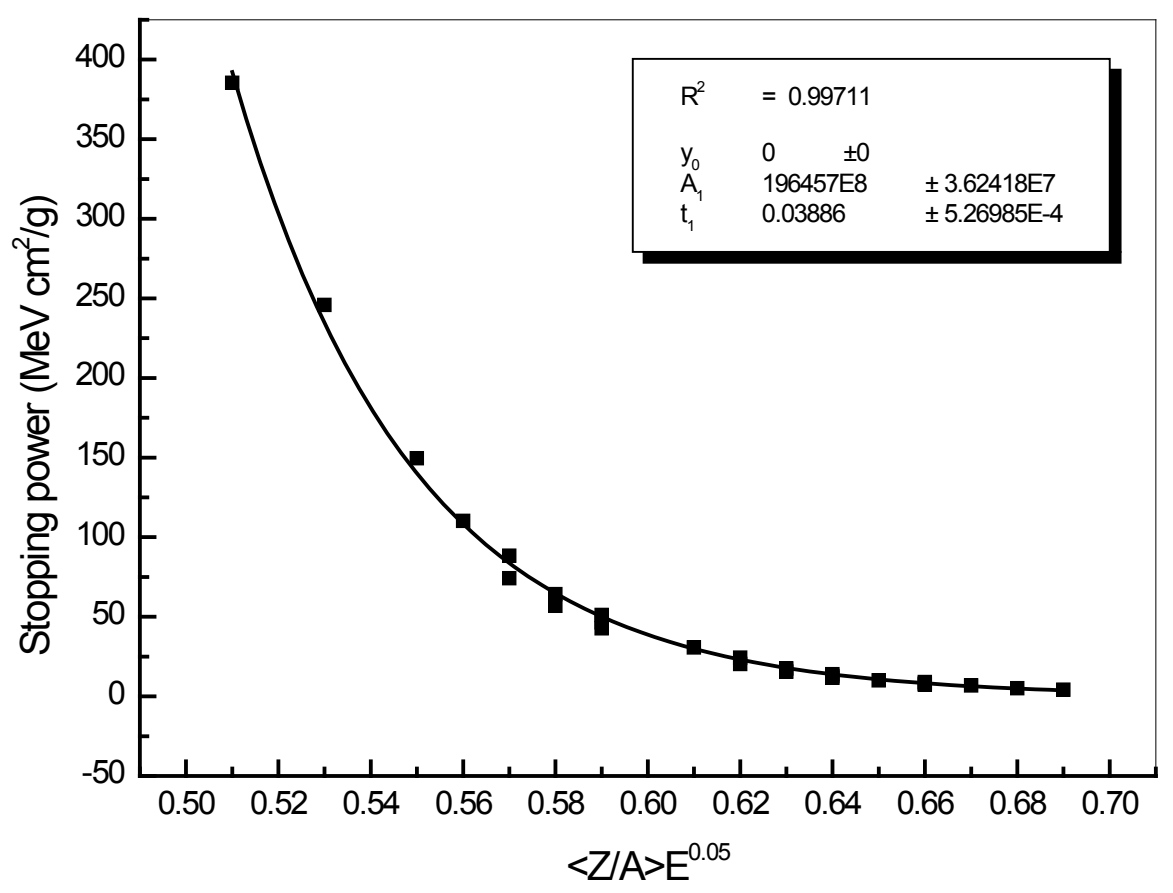


Figure 3. In this plot of mass stopping power (for protons) and $\left\langle\frac{Z}{A}\right\rangle E^{0.05}$ of Bone lie on a decreasing exponentially line. In this figure all values are taken from Ref. [2].

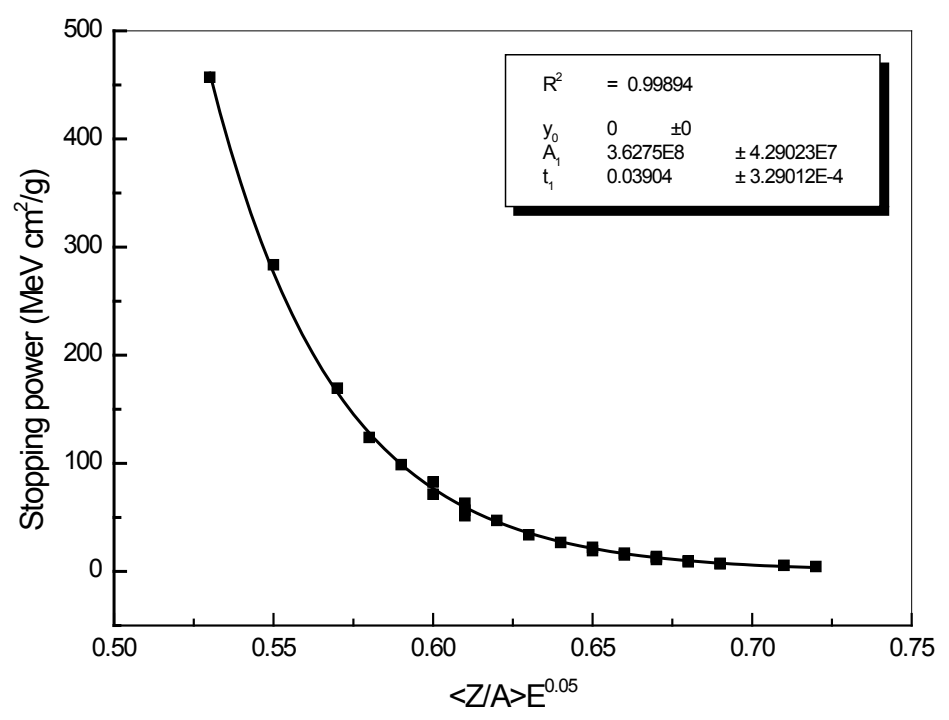
Figure 4. In this plot of mass stopping power (for protons) and $\left\langle\frac{Z}{A}\right\rangle E^{0.05} \quad$ of Tissue lie on a decreasing exponentially line. In this figure all values
are taken from Ref. [2]. 
Table 2. Values of stopping power (in $\mathrm{MeV} \mathrm{cm}^{2} / \mathrm{g}$ ) of water, muscle, bone and tissue

\begin{tabular}{|c|c|c|c|c|c|c|c|c|c|c|c|c|}
\hline $\begin{array}{l}\text { Energy } \\
(\mathrm{MeV})\end{array}$ & Water [2] & This work & $\%$ error & Muscle [2] & This work & $\%$ error & Bone [2] & This work & $\%$ error & Tissue [2] & This work & $\%$ error \\
\hline 0.5 & 446.02 & 439.19 & 1.5 & 431.71 & 422.06 & 2.2 & 385.45 & 373.10 & 3.2 & 457.08 & 446.31 & 2.4 \\
\hline 1 & 279.13 & 271.99 & 2.6 & 270.97 & 262.97 & 3.0 & 245.82 & 234.45 & 4.6 & 283.67 & 276.20 & 2.6 \\
\hline 2 & 167.77 & 165.62 & 1.3 & 163.19 & 161.14 & 1.3 & 149.59 & 144.93 & 3.1 & 169.54 & 168.06 & 0.9 \\
\hline 3 & 122.94 & 122.91 & 0.0 & 119.68 & 120.03 & 0.3 & 110.22 & 108.54 & 1.5 & 123.93 & 124.66 & 0.6 \\
\hline 4 & 98.16 & 99.10 & 1.0 & 95.61 & 97.05 & 1.5 & 88.29 & 88.09 & 0.2 & 98.8 & 100.48 & 1.7 \\
\hline 5 & 82.25 & 83.68 & 1.7 & 80.15 & 82.12 & 2.5 & 74.15 & 74.76 & 0.8 & 82.7 & 84.82 & 2.6 \\
\hline 6 & 71.11 & 72.78 & 2.3 & 69.31 & 71.54 & 3.2 & 64.23 & 65.30 & 1.7 & 71.44 & 73.75 & 3.2 \\
\hline 7 & 62.82 & 64.61 & 2.8 & 61.25 & 63.61 & 3.9 & 56.81 & 58.18 & 2.4 & 63.08 & 65.46 & 3.8 \\
\hline 8 & 56.41 & 58.23 & 3.2 & 55 & 57.41 & 4.4 & 51.07 & 52.61 & 3.0 & 56.6 & 59.00 & 4.2 \\
\hline 9 & 51.28 & 53.11 & 3.6 & 50 & 52.42 & 4.8 & 46.46 & 48.11 & 3.6 & 51.43 & 53.79 & 4.6 \\
\hline 10 & 47.07 & 48.88 & 3.8 & 45.91 & 48.30 & 5.2 & 42.69 & 44.39 & 4.0 & 47.2 & 49.51 & 4.9 \\
\hline 15 & 33.82 & 35.38 & 4.6 & 33 & 35.10 & 6.4 & 30.76 & 32.45 & 5.5 & 33.87 & 35.82 & 5.7 \\
\hline 20 & 26.73 & 28.02 & 4.8 & 26.09 & 27.88 & 6.8 & 24.35 & 25.88 & 6.3 & 26.74 & 28.35 & 6.0 \\
\hline 25 & 22.28 & 23.32 & 4.7 & 21.75 & 23.26 & 6.9 & 20.32 & 21.66 & 6.6 & 22.27 & 23.60 & 6.0 \\
\hline 30 & 19.2 & 20.05 & 4.4 & 18.75 & 20.03 & 6.8 & 17.53 & 18.71 & 6.7 & 19.19 & 20.28 & 5.7 \\
\hline \multicolumn{13}{|c|}{ Table 2 Continued......... } \\
\hline 35 & 16.94 & 17.62 & 4.0 & 16.55 & 17.64 & 6.6 & 15.48 & 16.51 & 6.6 & 16.93 & 17.82 & 5.3 \\
\hline 40 & 15.21 & 15.75 & 3.5 & 14.86 & 15.78 & 6.2 & 13.91 & 14.80 & 6.4 & 15.19 & 15.92 & 4.8 \\
\hline 45 & 13.84 & 14.25 & 2.9 & 13.51 & 14.30 & 5.8 & 12.66 & 13.43 & 6.1 & 13.82 & 14.40 & 4.2 \\
\hline 50 & 12.72 & 13.02 & 2.4 & 12.42 & 13.08 & 5.4 & 11.64 & 12.31 & 5.8 & 12.7 & 13.16 & 3.7 \\
\hline 60 & 11.01 & 11.14 & 1.1 & 10.756 & 11.21 & 4.2 & 10.085 & 10.58 & 4.9 & 10.987 & 11.25 & 2.4 \\
\hline 70 & 9.76 & 9.74 & 0.2 & 9.535 & 9.82 & 3.0 & 8.946 & 9.29 & 3.9 & 9.737 & 9.84 & 1.1 \\
\hline 80 & 8.8 & 8.67 & 1.5 & 8.602 & 8.76 & 1.8 & 8.074 & 8.30 & 2.8 & 8.781 & 8.76 & 0.2 \\
\hline 90 & 8 & 7.82 & 2.3 & 7.865 & 7.91 & 0.5 & 7.385 & 7.51 & 1.7 & 7.027 & 7.90 & 12.4 \\
\hline 100 & 7.437 & 7.12 & 4.2 & 7.267 & 7.21 & 0.7 & 6.826 & 6.86 & 0.5 & 7.415 & 7.19 & 3.0 \\
\hline 150 & 5.552 & 4.96 & 10.7 & 5.426 & 5.04 & 7.1 & 5.102 & 4.83 & 5.4 & 5.532 & 5.00 & 9.6 \\
\hline 200 & 4.579 & 3.82 & 16.7 & 4.476 & 3.89 & 13.0 & 4.212 & 3.74 & 11.1 & 4.56 & 3.85 & 15.6 \\
\hline
\end{tabular}




\section{Summary and Conclusions}

From the above results obtained using the proposed empirical relation (6), it is quite obvious that the stopping power of materials can be expressed in terms of energy and atomic number of the material. It is also noteworthy that proposed empirical relation is simpler, widely applicable and values obtained are in better agreement with the experimental and theoretical data as compared to the empirical relations proposed by previous researchers.

\section{REFERENCES}

[1] Raju M R. Heavy particle radiotherapy: Academic, New York; 1980.

[2] Mukherjee B. Mass stopping powers of protons up to 200 $\mathrm{MeV}$ in some biologically important materials. Nucl. Inst. Meth. 1983; 211: 235-237.

[3] Gauvin R. X -ray microanalysis of real materials using Monte Carlo simulations. Surf. Interface Anal. 2005;37:875.

[4] Ritchie N W M. A new Monte Carlo application for complex sample geometries.Surf. Interface Anal. 2005; 37: 1006.

[5] Salvat F, Llovet X,Fernandez-Varea J M and Sempau J. Monte Carlo simulation of X-ray emission using the general-purpose code PENELOPE. Surf. Interface Anal. 2005; 37: 1054.

[6] Bethe H. Zur Theorie des Durchgangs schneller Korpuskularstrahlen durch Materie Ann. Phys., 1930;5: 325.
[7] Bethe H A and Ashkin, J. Experimental Nuclear Physics, edited by E.Segre; Wiley, New York, 1953:166.

[8] Bethe H. Handbuch der Physik, edited by H. Geiger and K. Scheel: Springer, Berlin. 1933; 24(1): 273.

[9] Berger M J, Coursey J S, Zucker M A and Chang J. Stopping Power and Range Tables for Electrons, Positrons, and Helium Ions. 2005 (http://physics.nist.gov/PhysRefData/Star/Text/co ntents.html).

[10] Sargent B W. Procedure for calculating the radiation stopping power, Trans. Roy. Soc. Canada. 1928; 22(3): 179.

[11] Heitler W. (1936). The Quantum Theory of Radiation: Oxford Univ. Press, London. 1936: 220.

[12] Batra,R K and Sehgal M L. Empirical relation for total stopping power of positrons and electrons. Nuclear Physics A. 1970;156: 314 .

[13] Batra, R K and Sehgal M L. Approximate stopping power law of electrons and Positrons. Nucl. Inst Methods. 1973;109: 565.

[14] Batra,R K and Sehgal M L. Empirical relation for total stopping power of positons and electrons. Nuclear Physics A. 1972; 196: 638.

[15] Pal P B, Varshney V P and Gupta D K. Total Stopping Power Formulae for High Energy Electrons and Positrons. Nucl. Instru. Meth, B. 1986; 16: 1.

[16] Tanuma S, Powell C J and Penn D R. Calculations of stopping powers of $100 \mathrm{eV}-30 \mathrm{keV}$ electrons in 31 elemental solids. J. Appl. Phys. 2008; 063707: 103.

[17] Tan D and Heaton B. Simple empirical relations for electron CSDA range and electron energy loss, Applied Radiation and Isotope. 1994; 45: 527. 